\title{
Successful immediate cryorecanalization of a simultaneous high-grade tracheal and bronchial stenosis as rare manifestations of bronchial-associated lymphoid tissue lymphoma
}

\author{
Christian Schumann, MD, ${ }^{\mathrm{a}}$ Philipp M. Lepper, MD, ${ }^{\mathrm{a}, \mathrm{b}}$ Thomas F. E. Barth, MD, ${ }^{\mathrm{c}}$ Peter Möller, MD, ${ }^{\mathrm{c}}$ and Stefan Krüger, MD, ${ }^{\mathrm{a}}$ \\ Ulm, Germany, and Berne, Switzerland
}

Endobronchial tumor obstruction of central airways causes severe limitations for the patient. For these conditions, an effective and safe bronchoscopic treatment modality is required to ameliorate obstructive symptoms. Most physicians use mechanical debulking by the rigid scope, but neodymium-doped yttrium aluminium garnet (Nd:YAG) laser, argon plasma coagulation, and cryorecanalization are alternative techniques. ${ }^{1,2}$ The use of the flexible cryoprobe for immediate ablation of endoluminal tumors in lung cancer has been described earlier. ${ }^{3}$ This case reports for the first time the successful cryorecanalization of a combined severe tracheal and bronchial stenosis owing to bronchialassociated lymphoid tissue (BALT) lymphoma.

\section{CLINICAL SUMMARY}

A 70-year-old nonsmoking woman had persisting dry cough and exercise-induced dyspnea. Her general practitioner suspected asthma and treated her with inhaled betasympathomimetics and corticosteroids. As symptoms failed to improve, treatment was intensified and systemic corticosteroids were given over 4 months. After failure of this therapy, the patient was referred to a pulmonologist for further investigation. Clinical examination revealed a severe biphasic, predominantly inspiratory stridor, suggesting central airway stenosis. Subsequent lung function testing showed a nonreversible severe airway obstruction with markedly decreased peak expiratory flow (Figure 1, $A$ and $B$ ). Chest computed tomographic scan demonstrated a tracheal mass and a tumorous impression of the right main bronchus (Figure 1, C and $D$ ). Examination with a flexible bronchoscope confirmed severe midtracheal stenosis by an exophytic tumor extending to the proximal right main bronchus (Figure 1, $E$ and $F$ ).

For the ablation of the tumor, a flexible cryoprobe was used (Erbe Inc, Tuebingen, Germany). The patient was

\footnotetext{
From the Departments of Internal Medicine $\mathrm{II}^{\mathrm{a}}$ and Pathology, ${ }^{\mathrm{c}}$ University of Ulm, Ulm, Germany, and the Department of Intensive Care Medicine, ${ }^{\mathrm{b}}$ University Hospital of Berne (Inselspital), Berne, Switzerland.

Received for publication Jan 20, 2008; accepted for publication Feb 13, 2008.

Address for reprints: Dr med Christian Schumann, Zentrum für Innere Medizin,

Abteilung Innere Medizin II, Sektion Pneumologie, Universitätsklinikum Ulm,

Robert Koch Strasse 8, D-89081 Ulm, Germany (E-mail: christian.schumann@

uniklinik-ulm.de).

J Thorac Cardiovasc Surg 2009;137:e17-9

$0022-5223 / \$ 36.00$

Copyright (c) 2009 by The American Association for Thoracic Surgery

doi:10.1016/j.jtcvs.2008.02.043
}

sedated with intravenous disoprivan $(20 \mathrm{mg}$ bolus and $4 \mathrm{mg} \cdot \mathrm{kg}^{-1} \cdot \mathrm{h}^{-1}$ ) and intubated with a flexible endotracheal tube (Bronchoflex, $7.5 \mathrm{~mm}$; Rüsch $\mathrm{GmbH}$, Kernen, Germany). The endotracheal tube was placed directly above to the tracheal stenosis. During the tumor ablation the patient breathed spontaneously. Oxygen saturation, electrocardiogram, and blood pressure were monitored continuously. After contact of the probe's tip with the tumor, freezing was initiated for up to 10 seconds under full visual control of the operating physician. The frozen tumor was adherent to the tip of the cryoprobe and was gently pulled out through the tube. This procedure was repeated 6 times until the tumor in the trachea and the right main bronchus was nearly completely removed. After the procedure, the patient was immediately free from stridor and dyspnea. No severe bleeding or any other complications occurred. A second-look bronchoscopy was not necessary. Lung function testing on the following day confirmed significant improvements with decreased airway resistance and normalization of the flow-volume loop as well as the resistance loop. Histopathologic examination of the obtained tumor specimens showed BALT lymphoma in both locations (Figure $2, A$ to $C$ ). BALT lymphomas are known as rare extranodal manifestations of marginal zone B-cell lymphoma of mucosa-associated lymphoid tissue. ${ }^{4}$ Further examinations showed no peripheral blood or bone marrow involvement. The patient was treated with oral corticosteroids (40 mg dexamethasone for 3 days and subsequent tapering over 2 weeks). The clinical follow-up every 6 months remained unremarkable over 18 months without any signs of a relapse of the disease.

In a single session, the tumor was successfully removed from both locations without any complications.

\section{DISCUSSION}

BALT lymphomas of the trachea are rare. Depending on the location and size of the tumor, patients have severe dyspnea. An evidence-based management is not defined. Treatment options include complete surgical resection, radiation, or chemotherapy. As the primary treatment modality, chemotherapy including rituximab seems to be most effective. ${ }^{5}$

In the present case, the patient had predominantly inspiratory stridor and severe dyspnea owing to tumorous obstruction of the trachea and right main bronchus. For tumor removal, mechanical debulking by both a rigid scope and 

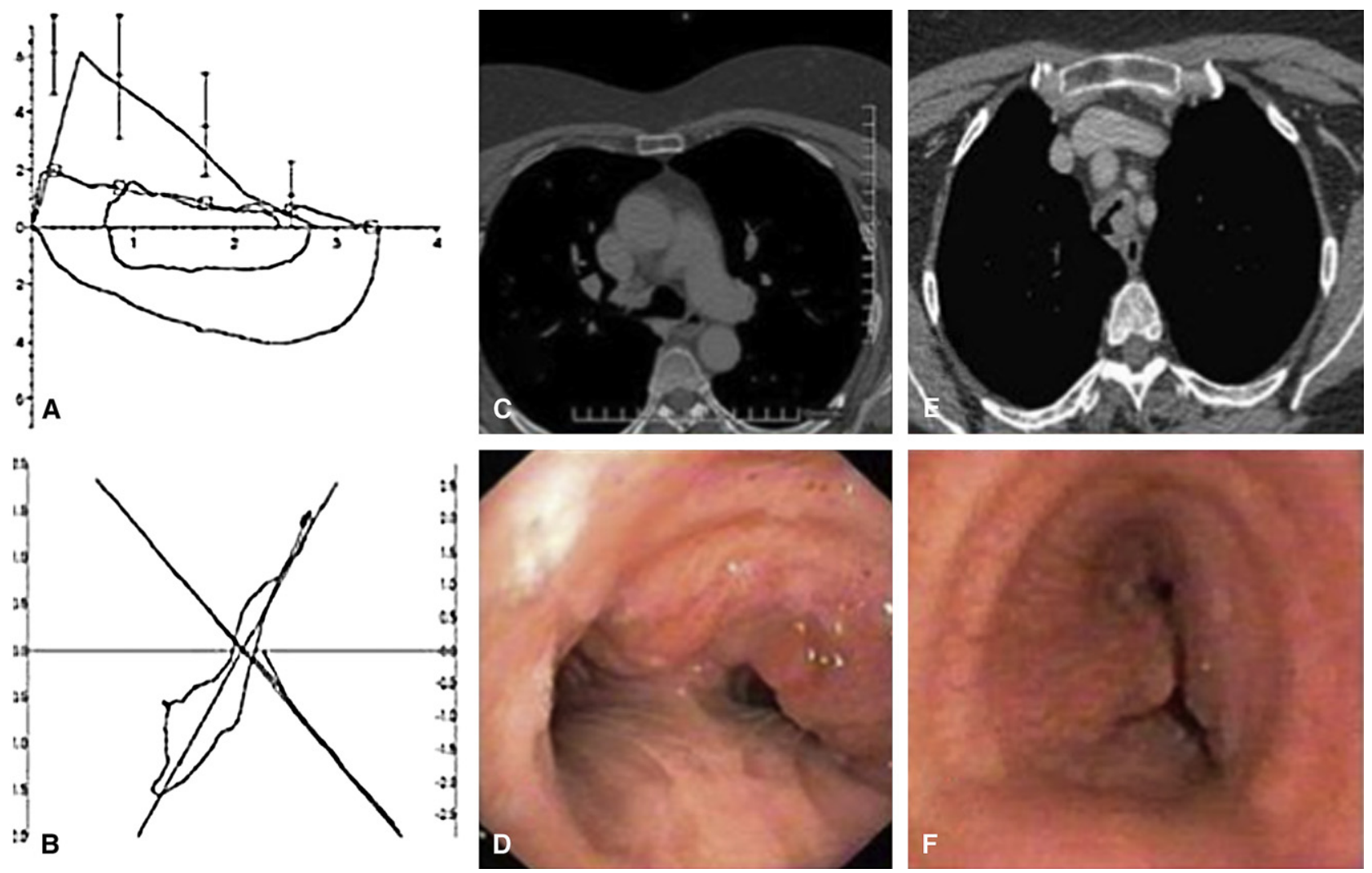

FIGURE 1. Lung function, chest computed tomographic scan, and bronchoscopic view of the stenosis. A and B, Flow-volume loop shows decreased peak expiratory flow and body plethysmography shows an abnormal resistance loop. $\mathrm{C}$ and $\mathrm{D}$, Axial view of chest computed tomographic scan shows high-grade midtracheal stenosis and midgrade stenosis of the right main bronchus. E and F, Bronchoscopy reveales tumorous manifestations in the trachea and the right main bronchus.

Nd:YAG laser, if required, combined with argon plasma coagulation are generally applicable. Cryorecanalization with the flexible cryoprobe is another complementary method for immediate management of airway obstruction by tumors. The cryoprobe can be used in full flexible technique as well as in combination with a rigid bronchoscope. The physician has visual control during the freezing process of the intervention. With this technique, we successfully treated both obstructing lesions within one bronchoscopic session.

Severe bleeding during recanalization with the cryoprobe is very uncommon; over a 5-year experience with this technique, we have never seen perforations, and the primary
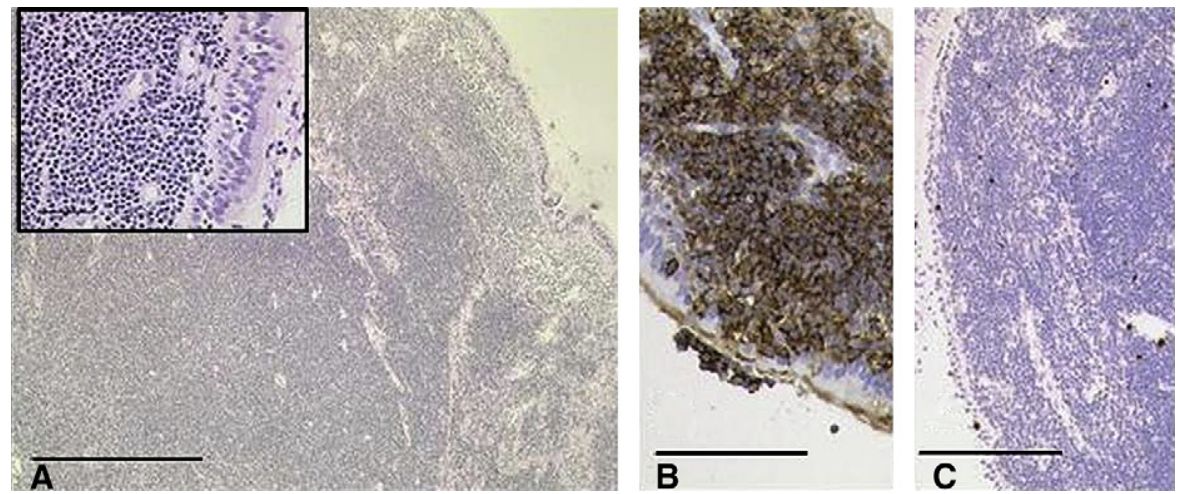

FIGURE 2. Morphology and immunohistochemistry of the BALT lymphoma. A, The lymphoma shows a diffuse growth pattern within the bronchus mucosa $($ bar $=500 \mu \mathrm{m}$ ) and consists of small lymphoma cells (see inset: $b a r=50 \mu \mathrm{m}$ ). B, The lymphoma cells are strongly CD20-positive and are also visible within the respiratory epithelium ( $b a r=100 \mu \mathrm{m})$. C: The proliferation rate is low. Ki-67 staining marked only some nuclei $($ bar $=200 \mu \mathrm{m})$. 
success rate of cryorecanalization is very high $(90 \%)$. Thus cryorecanalization should be taken into account for a safe and fast treatment of endoluminal airway stenoses such as tracheal and bronchial manifestations of BALT lymphoma.

\section{References}

1. Bolliger CT, Sutedja TG, Strausz J, Freitag L. Therapeutic bronchoscopy with immediate effect: laser, electrocautery, argon plasma coagulation and stents. Eur Respir J. 2006;27:1258-71.
2. Vergnon JM, Huber RM, Moghissi K. Place of cryotherapy, brachytherapy and photodynamic therapy in therapeutic bronchoscopy of lung cancers. Eur Respir J. 2006;28:200-18.

3. Hetzel M, Hetzel J, Schumann C, Marx N, Babiak A. Cryorecanalization: a new approach for the immediate management of acute airway obstruction. $J$ Thorac Cardiovasc Surg. 2004;127:1427-31.

4. Kuper CF. Histopathology of mucosa-associated lymphoid tissue. Toxicol Pathol. 2006;34:609-15.

5. Ahmed S, Kussick SJ, Siddiqui AK, Bhuiya TA, Khan A, Sarewitz S, et al. Bronchial-associated lymphoid tissue lymphoma: a clinical study of a rare disease. Eur $J$ Cancer. 2004;40:1320-6.

\section{New-onset heart failure caused by spontaneous papillary muscle rupture: Diagnosis with dual-source computed tomographic coronary angiography}

Heon Lee, MD, PhD, ${ }^{\mathrm{a}, \mathrm{d}}$ Balazs Ruzsics, MD, PhD, ${ }^{\mathrm{a}}$ U. Joseph Schoepf, MD, ${ }^{\mathrm{a}, \mathrm{b}}$ Edward A. Stewart, DO, ${ }^{\mathrm{b}}$ Philip Costello, MD, ${ }^{\mathrm{a}}$ James L. Glenn, MD, ${ }^{\mathrm{b}}$ and John S. Ikonomidis, $\mathrm{MD}, \mathrm{PhD},{ }^{\mathrm{c}}$ Charleston, SC, and Seoul, Republic of Korea

Papillary muscle rupture is a rare cardiac emergency most frequently associated with acute myocardial infarction or blunt chest trauma. ${ }^{1,2}$ Once papillary muscle rupture has been diagnosed, urgent mitral valve replacement is indicated to correct the patient's hemodynamic deterioration. Here we present the case of a patient with spontaneous, complete posterior papillary muscle rupture in the absence of significant coronary artery disease, diagnosed with dual-source computed tomographic (CT) coronary angiography. To our knowledge, this is the first reported establishment of this diagnosis with cardiac CT.

\section{CLINICAL SUMMARY}

A 49-year-old woman with a history of hypertension and hyperlipidemia was admitted to the general medical service with a 1-month history of progressive cough, orthopnea, dyspnea on exertion, and increasingly limited exercise ability. Severe mitral valve regurgitation was found on echocardiography. Right and left heart catheterizations showed mild, nonobstructive coronary artery disease. In addition, severe mitral regurgitation and an elevated left ventricular filling pressure were confirmed, with moderate pulmonary

\footnotetext{
From the Department of Radiology, ${ }^{a}$ the Division of Cardiology, ${ }^{b}$ and the Department of Surgery, ${ }^{\mathrm{c}}$ Medical University of South Carolina, Charleston, SC, and the Department of Radiology, Seoul Medical Center, Seoul, Republic of Korea. ${ }^{d}$

Received for publication Jan 23, 2008; accepted for publication Feb 28, 2008.

Address for reprints: U. Joseph Schoepf, MD, Department of Radiology, Medical University of South Carolina, 169 Ashley Ave, Charleston, SC 29425 (E-mail: schoepf@musc.edu).

J Thorac Cardiovasc Surg 2009;137:e19-21

0022-5223/\$36.00

Copyright (c) 2009 by The American Association for Thoracic Surgery

doi:10.1016/j.jtcvs.2008.02.054
}

hypertension. On the basis of these findings, mitral valve replacement was recommended. A contrast-enhanced cardiac CT scan was scheduled to assess overall cardiac anatomy before valve replacement surgery and to evaluate further the etiology of the new-onset heart failure. ${ }^{3}$

Retrospectively electrocardiographically gated CT coronary angiography was performed with a dual-source CT scanner (Definition; Siemens, Forchheim, Germany) with a temporal resolution of $83 \mathrm{~ms}$ and $0.6 \times 32 \times 2$ collimation. At the time of the CT scan acquisition, the patient was in tachyarrhythmia, with heart rates ranging from 115 to 127 beats/min. Such heart rates would not have allowed diagnostic imaging of the heart with slower, previous generation 16or 64-slice CT scanners. In accordance with institutional policy, no pharmacologic rate control was used. The scan was enhanced with $75 \mathrm{~mL}$ iopamidol contrast medium (Isovue $370 \mathrm{mgI} / \mathrm{mL}$; Bracco, Princeton, NJ) injected at $6 \mathrm{~mL} / \mathrm{s}$. Multiphase axial reconstruction was performed with 0.75 mm section thickness for morphologic and functional analyses with multiplanar reformations, 3-dimensional volume rendering, and cineloop display.

CT coronary angiography showed nonobstructive stenosis in the mid left anterior descending coronary artery in good correlation with previous coronary catheterization. The left atrium and ventricle were enlarged, with left ventricular hypertrophy compatible with hypertensive heart disease. Lung reconstructions showed moderate pulmonary edema and pleural effusions. Image reconstruction during diastole and systole (Figure 1) showed a low-attenuation mass, inferior to the posterior leaflet of the mitral valve in the expected location of the posterior papillary muscle. On 